International Journal of Pure and Applied Mathematics

Volume 99 No. 2 2015, 123-143

ISSN: 1311-8080 (printed version); ISSN: 1314-3395 (on-line version)

url: http://www.ijpam.eu

doi: http://dx.doi.org/10.12732/ijpam.v99i2.1

ijpam.eu

\title{
FINITE ELEMENT ANALYSIS OF UNSTEADY RADIATIVE MHD NATURAL CONVECTION COUETTE FLOW BETWEEN PERMEABLE PLATES WITH VISCOUS AND JOULE DISSIPATION
}

\author{
Victor M. Job ${ }^{1}$, Sreedhara Rao Gunakala ${ }^{\S}$ \\ 1,2 Department of Mathematics and Statistics \\ The University of West Indies \\ St. Augustine, TRINIDAD AND TOBAGO
}

\begin{abstract}
This paper discusses the unsteady magnetohydrodynamic free convection Couette flow of an incompressible viscous fluid between two infinite vertical permeable plates in the presence of thermal radiation with an exponentially decaying pressure gradient. A uniform magnetic field that is perpendicular to the plates, and uniform suction and injection through the plates are applied. The magnetic field lines are assumed to be fixed relative to the moving plate. The momentum equation takes buoyancy forces into consideration, while the energy equation considers thermal radiation effects and viscous and Joule dissipations. The fluid is considered to be a gray absorbing-emitting but nonscattering medium in the optically thick limit. The Rosseland approximation is used to describe the radiative heat flux in the energy equation. The coupled pair of non-linear partial differential equations is discretized using the Galerkin finite element method. This yields a system of non-linear algebraic equations which is solved using an iterative method to obtain the velocity and temperature distributions. The effects of suction parameter $\mathrm{S}$, radiation parameter $R_{d}$,
\end{abstract}

Received: May 15, 2014

(c) 2015 Academic Publications, Ltd. url: www.acadpubl.eu

$\S_{\text {Correspondence author }}$ 
Grashof number Gr, magnetic parameter H, Prandtl number Pr and Eckert number $E_{c}$ on the velocity and temperature distributions are investigated.

AMS Subject Classification: 76R10, 76W05

Key Words: MHD, natural convection, thermal radiation, couette flow, permeable plates, finite element method

\section{Introduction}

Magnetohydrodynamics (MHD) is the study of electrically conducting fluids in the presence of an imposed magnetic field. The study of MHD is important due to its engineering and industrial applications such as the use of MHD pumps in chemical energy technology and MHD power generators [7]. Other applications of MHD include the electrolysis of aluminium oxide to aluminium and the heating, stirring and levitation of liquid metals [4]. The steady flow of an electrically conducting fluid between two infinite parallel stationary plates in the presence of a transverse uniform magnetic field was first studied by Hartmann and Lazarus in 1937 ([1], [18]). Rossow [18] considered the flow of an electrically conducting fluid over a flat plate in the presence of a transverse magnetic field in the cases where the magnetic field is fixed relative to the plate and fixed relative to the fluid. It was discovered that when the magnetic field is considered to be fixed relative to the plate, the flow is accelerated by the magnetic field. In the case where the magnetic field is fixed relative to the fluid, the fluid flow is retarded by the magnetic field.

Couette flow is an important flow phenomenon with respect to engineering applications involving shear-driven flow such as aerodynamic heating and polymer technology [3]. The study of MHD Couette flow is particularly useful in gaining a better understanding of electrostatic precipitation and the operation of MHD power generators [2]. Attia [2] considered the unsteady MHD Couette flow of a Newtonian fluid with suction and injection through the plates and viscous and Joule dissipations under a constant pressure gradient. The velocity and temperature distributions were obtained using the finite difference method. The results showed that the velocity decreases as the magnetic field strength increases. Increasing the strength of the magnetic field was shown to increase the temperature at small times but decrease the temperature at large times. It was also observed that increasing the suction parameter results in a decrease in both the velocity and temperature.

Natural (Free) convection is the motion of fluid which is induced by temperature differences within the fluid. The study of free convection flow is im- 
portant in geophysics, problems involving the spread of pollution and other energy-related engineering applications [16]. Natural convection in fluid flows has been investigated mathematically by many authors. The effect of natural convection on unsteady Couette flow was studied by Singh [19]. The Laplace transform technique was used to obtain the velocity and temperature fields, the skin-friction and rate of heat transfer. It was observed that an increase in the Grashof number results in an increase in the flow velocity. Jha [7] extended the work of Singh [19] by discussing the combined effects of natural convection and a uniform transverse magnetic field when the magnetic field is fixed relative to the plate or fluid. Using the Laplace transform technique, an exact solution was obtained for the velocity and temperature fields. The trends observed with respect to the magnetic parameter are consistent with those observed in Rossow [18]. Jha and Apere [8] extended the work of Jha [7] by considering the unsteady MHD free convection Couette flow between two vertical parallel porous plates with uniform suction and injection. The cases where the magnetic field is considered fixed relative to the fluid and fixed relative to the moving plate were considered. The velocity and temperature distributions were obtained using the Laplace transform technique. The results revealed that the temperature and velocity of the fluid decrease with increasing Prandtl number and with increasing suction/injection parameter.

The effect of thermal radiation in fluids is a subject of growing interest in the applied sciences, since thermal radiation has a significant impact on heat transfer in fluids encountered in engineering and industry. Thermal radiation effects in fluid flow are significant when the temperature of the fluid is high. Heat transfer in furnaces, engine combustion chambers, and power plants for gas cooled nuclear reactors is influenced considerably by thermal radiation effects [14]. In the limit of an optically thick medium, the Rosseland Approximation is commonly used to describe the relation between the radiative flux and the temperature of the medium [13]. Rajput and Sahu [15] investigated the unsteady natural convection hydromagnetic Couette flow between two infinite vertical plates in the presence of thermal radiation. The magnetic field was assumed to be fixed relative to the moving plate, and the cases of impulsive and uniformly accelerated movement of the plate were considered. The Rosseland approximation was used to describe the radiative heat flux. The velocity and temperature distributions, skin-friction and Nusselt number were obtained using the Laplace transform technique. The authors discovered that the velocity and temperature decrease with increasing Prandtl number and with increasing radiation parameter in both cases. Increasing the Grashof number results in an increase in flow velocity. It was also observed that increasing the magnetic 
parameter increases the velocity in the case of impulsive movement of the plate but decreases the velocity in the case of uniformly accelerated movement of the plate. Job and Gunakala [9] extended the work done by Rajput and Sahu [15] by including the effects of suction and injection through permeable plates. The velocity and temperature distributions were approximated using the finite element method. The work of Rajput and Sahu [15] was also extended by Job and Gunakala [10] by incorporating viscous and Joule dissipation effects using the finite element method. This was further extended by Job and Gunakala [11] by considering the presence of an exponentially decaying pressure gradient. It was observed that an increase in the radiation parameter and in the Prandtl number causes the fluid velocity and temperature to decrease at small time but increase at large time. The temperature of the fluid was shown to increase with increasing magnetic parameter. The velocity increases with increasing magnetic number in the case of impulsive movement of the plate. However, in the case of uniformly accelerated movement of the plate, an increase in the magnetic parameter results in a decrease in the flow velocity at small time and an increase in the flow velocity at large time. It was observed that in both cases, the Grashof number and Eckert number are proportional to both the flow velocity and fluid temperature.

This paper aims to further extend the work of Rajput and Sahu [15] by incorporating the effects of uniform suction and injection through permeable plates with viscous and Joule dissipations in the presence of an exponentially decaying pressure gradient.

\section{Formulation of the Problem}

Consider the flow of a Newtonian fluid between two parallel vertical nonconducting permeable plates. These plates are located on planes $y=0$ and $y=h$, and are infinite in the $x$ and $z$ directions. The plate at $y=h$ is stationary and the other plate moves with time-dependent velocity $U_{0} t^{c}$ in the positive $x$-direction (where $U_{0}$ is constant and $c$ is a non-negative integer). The temperature of the moving and stationary plates are fixed at $T_{1}$ and $T_{2}$ respectively, with $T_{1}>T_{2}$. An exponentially decaying pressure gradient $-\frac{\partial p}{\partial x}$ and a uniform magnetic field with magnitude $B_{0}$ are applied at $t=0$ in the positive $x$ and $y$ directions respectively. Uniform suction through the moving plate and uniform injection through the stationary plate are applied at $t=0$ in the negative $y$ direction.

We make the following assumptions: 


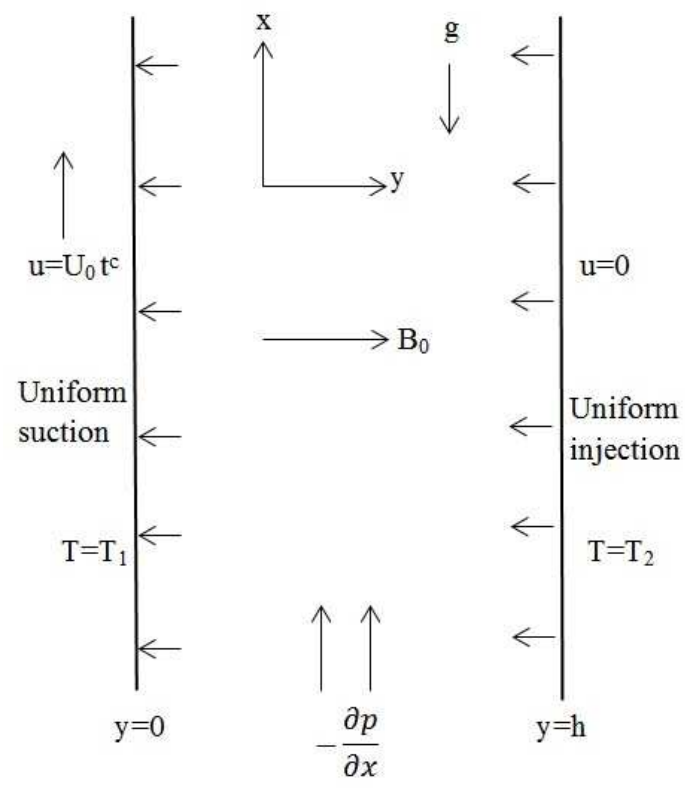

Figure 1: Schematic Diagram of the Physical System

1. The flow is incompressible, i.e., $\rho$ is constant.

2. The magnetic Reynolds number is very small.

3. For a typical conductor, $\rho_{e}$ is very small compared to the Lorentz force; hence it is negligible.

4. The Boussinesq approximation is applied.

5. The fluid is a gray and optically thick absorbing-emitting but non-scattering medium.

6. The fluid has a refractive index of unity.

With these assumptions, the governing equations are:

Continuity Equation:

$$
\frac{\partial u}{\partial x}=0
$$

Momentum Equation:

$$
\rho\left(\frac{\partial u}{\partial t}-v_{o} \frac{\partial u}{\partial y}\right)=-\frac{\partial p}{\partial x}+\rho g \alpha\left(T-T_{2}\right)-\gamma B_{0}^{2}\left(u-U_{0} t^{c}\right)+\mu \frac{\partial^{2} u}{\partial y^{2}}
$$


Energy Equation:

$$
\rho c_{p}\left(\frac{\partial T}{\partial t}-v_{0} \frac{\partial T}{\partial y}\right)=\kappa \frac{\partial^{2} T}{\partial y^{2}}-\frac{\partial q_{r}}{\partial y}+\mu\left(\frac{\partial u}{\partial y}\right)^{2}+\gamma B_{0}^{2} u^{2}
$$

where $u$ is the flow velocity in the $x$-direction, $\rho$ is the fluid density, $v_{0}$ is the constant suction/injection velocity, $p$ is the fluid pressure, $g$ is the acceleration due to gravity, $\alpha$ is the coefficient of thermal expansion, $T$ is the fluid temperature, $\gamma$ is the electrical conductivity, $\mu$ is the viscosity, $c_{p}$ is the specific heat capacity at constant pressure, $\kappa$ is the thermal conductivity and $q_{r}$ is the radiative heat flux. The initial and boundary conditions for the velocity and temperature are given by

$$
\begin{gathered}
u(y, 0)=0, u(0, t)=U_{0} t^{c}, u(h, t)=0 \\
T(y, 0)=T_{2}, T(0, t)=T_{1}, T(h, t)=T_{2}
\end{gathered}
$$

Using the Rosseland approximation, the radiative heat flux is given by

$$
q_{r}=-\frac{4 \sigma}{3 \beta_{R}} \frac{\partial T^{4}}{\partial y}
$$

where $\sigma$ is the Stefan-Boltzmann constant and $\beta_{R}$ is the mean absorption coefficient.

The temperature differences within the fluid are assumed to be small enough that $q_{r}$ may be considered a linear function of $T$ [15]. Thus, on taking a Taylor series expansion of $T^{4}$ about $T_{2}$ (up to first order approximation), we get

$$
T^{4} \cong T_{2}^{4}+4\left(T-T_{2}\right) T_{2}^{3}=4 T_{2}^{3} T-3 T_{2}^{4}
$$

Substituting this approximation into equation (6) gives

$$
q_{r}=-\frac{16 \sigma T_{2}^{3}}{3 \beta_{R}} \frac{\partial T}{\partial y}
$$

which on subtituting into equation (3) gives

$$
\rho c_{p}\left(\frac{\partial T}{\partial t}-v_{0} \frac{\partial T}{\partial y}\right)=\left(\kappa+\frac{16 \sigma T_{2}^{3}}{3 \beta_{R}}\right) \frac{\partial^{2} T}{\partial y^{2}}+\mu\left(\frac{\partial u}{\partial y}\right)^{2}+\gamma B_{0}^{2} u^{2}
$$

The following dimensionless variables are defined:

$$
\hat{x}=\frac{x}{h}, \hat{y}=\frac{y}{h}, \hat{u}=\frac{u}{U_{0}}\left(\frac{\mu}{\rho h^{2}}\right)^{c}, \hat{t}=\frac{t \mu}{\rho h^{2}}, \hat{p}=\frac{p h}{\mu U_{0}}\left(\frac{\mu}{\rho h^{2}}\right)^{c},
$$




$$
\begin{gathered}
\hat{T}=\frac{T-T_{2}}{T_{1}-T_{2}}, S=\frac{\rho v_{o} h}{\mu}, H=\frac{\gamma B_{0}^{2} h^{2}}{\mu}, G r=\frac{\rho g h^{2} \alpha\left(T_{1}-T_{2}\right)}{\mu U_{0}}\left(\frac{\mu}{\rho h^{2}}\right)^{c}, \\
\operatorname{Pr}=\frac{\mu c_{p}}{\kappa}, R_{d}=\frac{\kappa \beta_{R}}{4 \sigma T_{2}^{3}}, E_{c}=\frac{U_{0}^{2}}{c_{p}\left(T_{1}-T_{2}\right)}\left(\frac{\rho h^{2}}{\mu}\right)^{2 c}
\end{gathered}
$$

where $\mathrm{S}$ is the suction parameter, $\mathrm{H}$ is the magnetic parameter, $\mathrm{Gr}$ is the Grashof number, $\operatorname{Pr}$ is the Prandtl number, $R_{d}$ is the radiation parameter and $E_{c}$ is the Eckert number. On dropping the hat notation and taking the dimensionless pressure gradient to be $-\frac{\partial p}{\partial x}=C e^{-\beta t}$ for constants $C$ and $\beta$, equations (2) and (7) become

$$
\frac{\partial u}{\partial t}-S \frac{\partial u}{\partial y}=C e^{-\beta t}+G r T-H\left(u-t^{c}\right)+\frac{\partial^{2} u}{\partial y^{2}}
$$

and

$$
\frac{\partial T}{\partial t}-S \frac{\partial T}{\partial y}=\left(\frac{3 R_{d}+4}{3 R_{d} P r}\right) \frac{\partial^{2} T}{\partial y^{2}}+E_{c}\left(\frac{\partial u}{\partial y}\right)^{2}+H E_{c} u^{2}
$$

The initial and boundary conditions for the flow velocity are given by

$$
u(y, 0)=0, u(0, t)=t^{c}, u(1, t)=0
$$

and those for the fluid temperature are written as

$$
T(y, 0)=0, T(0, t)=1, T(1, t)=0
$$

\section{Finite Element Method}

\subsection{Spatial Semi-Discretization}

We discretize the spatial domain $(0,1)$ into elements $\Omega_{1}^{k}=\left(y_{a}^{k}, y_{b}^{k}\right)$ for $k=$ $1,2, . ., r$. The Lagrange quadratic interpolation basis functions $\psi_{1}^{k}, \psi_{2}^{k}$ and $\psi_{3}^{k}$ are chosen for the approximate solutions $u_{k}$ of $u$ and $T_{k}$ of $T$ over $\Omega_{1}^{k}$. Under Galerkin's finite element method, the weight functions are taken to be the $\psi_{i}^{k}$, for $i=1,2,3$. In accordance with the weighted integral formulation [17], we have

$$
\int_{\Omega_{1}^{k}}\left(\frac{\partial u_{k}}{\partial t}-S \frac{\partial u_{k}}{\partial y}-C e^{-\beta t}-G r T_{k}+H\left(u_{k}-t^{c}\right)-\frac{\partial^{2} u_{k}}{\partial y^{2}}\right) \psi_{i}^{k} d y=0
$$




$$
\int_{\Omega_{1}^{k}}\left(\frac{\partial T_{k}}{\partial t}-S \frac{\partial T_{k}}{\partial y}-\left(\frac{3 R_{d}+4}{3 R_{d} P r}\right) \frac{\partial^{2} T_{k}}{\partial y^{2}}-E_{c}\left(\frac{\partial u_{k}}{\partial y}\right)^{2}-H E_{c} u_{k}^{2}\right) \psi_{i}^{k} d y=0
$$

We take approximations of the form

$$
u_{k}(y, t)=\sum_{j=1}^{3} \xi_{j}^{k}(t) \psi_{j}^{k}(y), T_{k}(y, t)=\sum_{j=1}^{3} \eta_{j}^{k}(t) \psi_{j}^{k}(y)
$$

Using integration by parts, rearranging and substituting (15) into equations (13) and (14) yields the semi-discrete system

$$
\begin{gathered}
\sum_{j=1}^{3} A_{i j}^{k} \frac{d \xi_{j}^{k}}{d t}+\sum_{j=1}^{3} B_{i j}^{k 1} \xi_{j}^{k}+\sum_{j=1}^{3} B_{i j}^{k 2} \eta_{j}^{k}=Q_{i}^{k}+f_{i}^{k} \\
\sum_{j=1}^{3} A_{i j}^{k} \frac{d \eta_{j}^{k}}{d t}+\sum_{j=1}^{3} B_{i j}^{k 3} \xi_{j}^{k}+\sum_{j=1}^{3} B_{i j}^{k 4} \eta_{j}^{k}=R_{i}^{k}
\end{gathered}
$$

where

$$
\begin{gathered}
A_{i j}^{k}=\int_{y_{1}^{k}}^{y_{3}^{k}} \psi_{i}^{k} \psi_{j}^{k} d y, B_{i j}^{k 1}=\int_{y_{1}^{k}}^{y_{3}^{k}}\left(H \psi_{i}^{k} \psi_{j}^{k}-S \psi_{i}^{k} \frac{d \psi_{j}^{k}}{d y}+\frac{d \psi_{i}^{k}}{d y} \frac{d \psi_{j}^{k}}{d y}\right) d y \\
B_{i j}^{k 2}=\int_{y_{1}^{k}}^{y_{3}^{k}}-G r \psi_{i}^{k} \psi_{j}^{k} d y=-G r A_{i j}^{k} \\
B_{i j}^{k 3}=-E_{c} \int_{y_{1}^{k}}^{y_{3}^{k}} \psi_{i}^{k}\left(\frac{d \psi_{j}^{k}}{d y} \sum_{p=1}^{3} \xi_{p}^{k} \frac{d \psi_{p}^{k}}{d y}+H \psi_{j}^{k} \sum_{p=1}^{3} \xi_{p}^{k} \psi_{p}^{k}\right) d y \\
B_{i j}^{k 4}=\int_{y_{1}^{k}}^{y_{3}^{k}}\left(\left(\frac{3 R_{d}+4}{3 R_{d} P r}\right) \frac{d \psi_{i}^{k}}{d y} \frac{d \psi_{j}^{k}}{d y}-S \psi_{i}^{k} \frac{d \psi_{j}^{k}}{d y}\right) d y \\
f_{i}^{k}=\left(C e^{-\beta t}+H t^{c}\right) \int_{y_{1}^{k}}^{y_{3}^{k}} \psi_{i}^{k} d y, Q_{i}^{k}=\left[\psi_{i}^{k} \sum_{j=1}^{3} \frac{\xi_{j}^{k}}{d \psi_{j}^{k}}\right]_{y_{1}^{k}}
\end{gathered}
$$




$$
R_{i}^{k}=\left[\psi_{i}^{k} \sum_{j=1}^{3}\left(\frac{3 R_{d}+4}{3 R_{d} P r}\right) \eta_{j}^{k} \frac{d \psi_{j}^{k}}{d y}\right]_{y_{1}^{k}}^{y_{3}^{k}}
$$

\subsection{Time Discretization}

The time domain $(0, L)$ is discretized into elements $\Omega_{2}^{n}=\left(t_{n}, t_{n+1}\right)$ for $n=$ $1,2, . ., N-1$, where the Lagrange linear interpolation basis functions $\phi_{1}$ and $\phi_{2}$ are chosen for $\xi_{j}^{k}$ and $\eta_{j}^{k}$ respectively. Under the Galerkin finite element method, the weight functions are chosen to be the $\phi_{s}$, for $s=1,2$. Thus, the weighted integral forms of equations (16) and (17) are:

$$
\int_{\Omega_{2}^{n}}\left(\sum_{j=1}^{3} A_{i j}^{k} \frac{d \xi_{j}^{k}}{d t}+\sum_{j=1}^{3} B_{i j}^{k 1} \xi_{j}^{k}+\sum_{j=1}^{3} B_{i j}^{k 2} \eta_{j}^{k}-Q_{i}^{k}-f_{i}^{k}\right) \phi_{s} d t=0
$$

and

$$
\int_{\Omega_{2}^{n}}\left(\sum_{j=1}^{3} A_{i j}^{k} \frac{d \eta_{j}^{k}}{d t}+\sum_{j=1}^{3} B_{i j}^{k 3} \xi_{j}^{k}+\sum_{j=1}^{3} B_{i j}^{k 4} \eta_{j}^{k}-R_{i}^{k}\right) \phi_{s} d t=0
$$

The following forms are assumed:

$$
\begin{aligned}
\xi_{j}^{k}(t) & =\sum_{m=n}^{n+1} \xi_{j, m}^{k} \phi_{m-n+1}(t), \eta_{j}^{k}(t)=\sum_{m=n}^{n+1} \eta_{j, m}^{k} \phi_{m-n+1}(t) \\
f_{i}^{k}(t)=\sum_{m=n}^{n+1} f_{i, m}^{k} \phi_{m-n+1}(t), Q_{i}^{k}(t) & =\sum_{m=n}^{n+1} Q_{i, m}^{k} \phi_{m-n+1}(t) \\
R_{i}^{k}(t)=\sum_{m=n}^{n+1} R_{i, m}^{k} \phi_{m-n+1}(t), B_{i j}^{k 3}\left(\xi_{p}^{k}(t)\right) & =\sum_{m=n}^{n+1} B_{i j, m}^{k 3}\left(\xi_{p, m}^{k}\right) \phi_{m-n+1}(t)
\end{aligned}
$$

where $\xi_{j, m}^{k}=\xi_{j}^{k}\left(t_{m}\right), \eta_{j, m}^{k}=\eta_{j}^{k}\left(t_{m}\right), f_{i, m}^{k}=f_{i}^{k}\left(t_{m}\right), Q_{i, m}^{k}=Q_{i}^{k}\left(t_{m}\right), R_{i, m}^{k}=$ $R_{i}^{k}\left(t_{m}\right)$ and $B_{i j, m}^{k 3}\left(\xi_{p, m}^{k}\right)=B_{i j}^{k 3}\left(\xi_{p}^{k}\left(t_{m}\right)\right)$. On rearranging, substituting into equations (18) and (19) and taking $s=2$, the fully discretized element equations are obtained in the following matrix form:

$$
\begin{gathered}
{\left[\begin{array}{cc}
3 \mathbf{A}^{k}+2 \Delta \mathbf{B}^{k 1} & 2 \Delta \mathbf{B}^{k 2} \\
\Delta\left(\mathbf{B}_{n}^{k 3}+3 \mathbf{B}_{n+1}^{k 3}\right) & 6 \mathbf{A}^{k}+4 \Delta \mathbf{B}^{k 4}
\end{array}\right]\left[\begin{array}{c}
U_{n+1}^{k} \\
X_{n+1}^{k}
\end{array}\right]} \\
=\left[\begin{array}{cc}
3 \mathbf{A}^{k}-\Delta \mathbf{B}^{k 1} & -\Delta \mathbf{B}^{k 2} \\
-\Delta\left(\mathbf{B}_{n}^{k 3}+\mathbf{B}_{n+1}^{k 3}\right) & 6 \mathbf{A}^{k}-2 \Delta \mathbf{B}^{k 4}
\end{array}\right]\left[\begin{array}{c}
U_{n}^{k} \\
X_{n}^{k}
\end{array}\right]
\end{gathered}
$$




$$
+\Delta\left[\begin{array}{c}
F_{n}^{k}+Q_{n}^{k}+2\left(F_{n+1}^{k}+Q_{n+1}^{k}\right) \\
2 R_{n}^{k}+4 R_{n+1}^{k}
\end{array}\right]
$$

where $\mathbf{A}^{k}=\left(A_{i j}^{k}\right)$ is the local mass matrix, $\mathbf{B}^{k 1}=\left(B_{i j}^{k 1}\right), \mathbf{B}^{k 2}=\left(B_{i j}^{k 2}\right), \mathbf{B}_{n}^{k 3}=$ $\left(B_{i j, n}^{k 3}\right)$ and $\mathbf{B}^{k 4}=\left(B_{i j}^{k 4}\right)$ are the local stiffness matrices, $U_{n}^{k}=\left(\xi_{j, n}^{k}\right)$ is the local vector of velocity coefficients, $X_{n}^{k}=\left(\eta_{j, n}^{k}\right)$ is the local vector of temperature coefficients, and $F_{n}^{k}+Q_{n}^{k}=\left(f_{i, n}^{k}+Q_{i, n}^{k}\right)$ and $R_{n}^{k}=\left(R_{i, n}^{k}\right)$ are the local force vectors.

For computation of the solution, the spatial domain is divided into 40 line elements with uniform step-size $l$, while the time domain is divided into 500 line elements with uniform step-size $\Delta$. After assembly of the elements and applying the given boundary conditions, a system of 162 non-linear equations is obtained at each time level. Using an iterative method, an approximate solution for this system of equations is obtained to within an accuracy of $5 \times 10^{-4}$.

\section{Results and Discussion}

The approximate solutions for the fluid velocity and temperature were computed for different values of the suction parameter, radiation parameter, Grashof number, magnetic parameter, Prandtl number and Eckert number in the cases of impulsive $(c=0)$ and uniformly accelerated $(c=1)$ movement of the plate at $y=0$. The following values of the above parameters were used in the analysis of the numerical results: suction parameter $S=1,3,5$; radiation parameter $R_{d}=0.1,1,10$; Grashof number $G r=1,5,10$; magnetic parameter $H=2,4,6$; Prandtl number $\operatorname{Pr}=0.71$ (for air), 3 (for the saturated liquid Freon at $273.3 \mathrm{~K}$ ), 7 (for water) and Eckert number $E_{c}=0.2,1,2$. Throughout the analysis, the parameters $C$ and $\beta$ are taken to be 5 and 1.2 respectively.

The velocity and temperature profiles for the current finite element solution with $\mathrm{S}, E_{c}$, C and $\beta$ set to zero was compared with the previous results of Rajput and Sahu [15] in Figures 2 and 3. It was observed that the present numerical results are in excellent agreement with that obtained in [15].

Figures 4-7 show the effects of the suction parameter $\mathrm{S}$ on the time development of the velocity and temperature of the fluid at the centre of the channel $(y=0.5)$. It was observed that both the fluid velocity and temperature decrease with increasing suction parameter. The observed trend for the suction parameter may be attributed to the suction and injection through the plates, which effect a transfer of fluid near the stationary plate (which has lower velocity) to the centre of the channel (which has higher velocity). This, in turn, causes the flow velocity at the centre of the channel to decrease. Since the fluid at the 


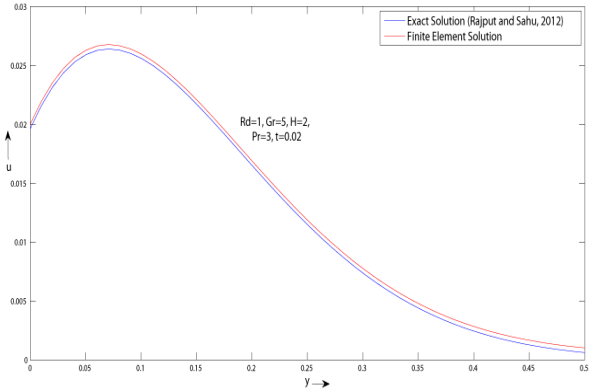

Figure 2: Comparison of Velocity Profiles in the Case of Uniformly Accelerated Movement of the Plate

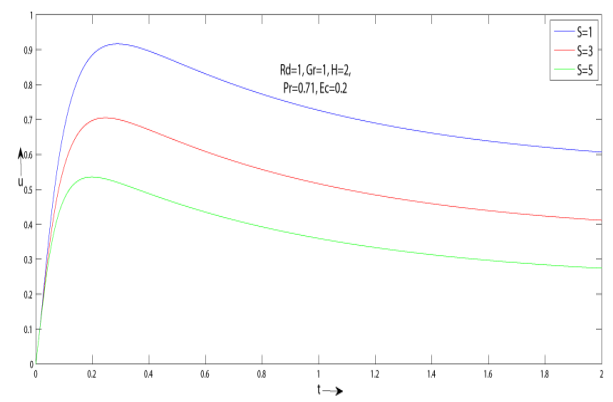

Figure 4: Time Development of Velocity for Different Values of S in the Case of Impulsive Movement of the Plate

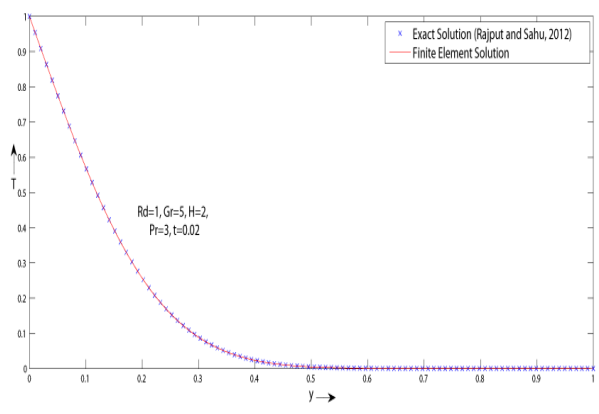

Figure 3: Comparison of Temperature Profiles in the Case of Uniformly Accelerated Movement of the Plate

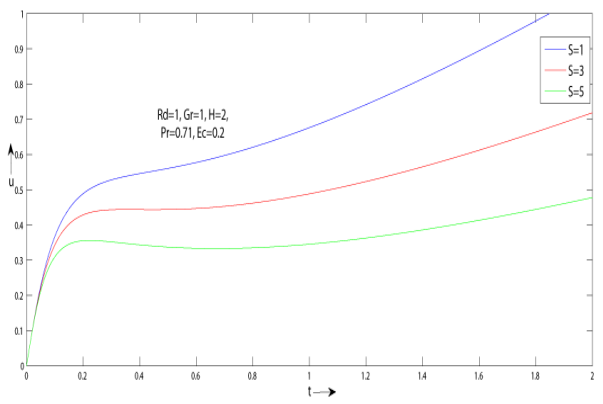

Figure 5: Time Development of Velocity for Different Values of S in the Case of Uniformly Accelerated Movement of the Plate

centre of the channel has a higher temperature than the fluid which is near to the stationary plate, the fluid transfer due to suction and injection results in a decrease in fluid temperature at the centre of the channel.

The effects of the Grashof number Gr on the velocity and temperature of the fluid at the centre of the channel $(y=0.5)$ are displayed in figures 8-11. The results show that both the velocity and temperature of the fluid increase with increasing Gr. Since the Grashof number is proportional to the bouyancy forces associated with free convection within the fluid, an increase in Gr increases free convection effects within the fluid, which results in an increase in the flow velocity as observed in figures 8 and 9 . The increase in fluid temperature 


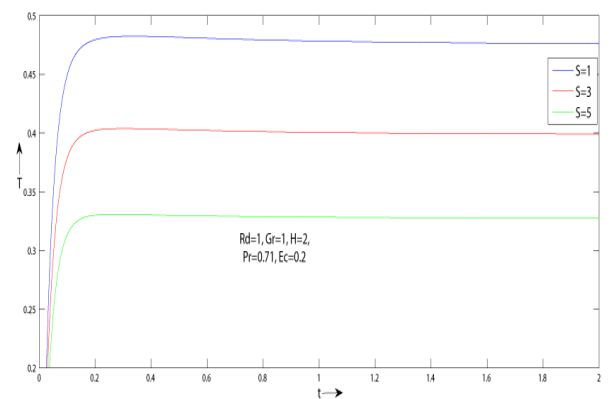

Figure 6: Time Development of Temperature for Different Values of $\mathrm{S}$ in the Case of Impulsive Movement of the Plate

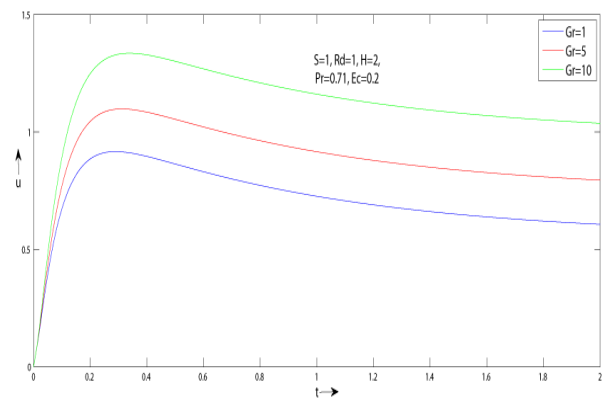

Figure 8: Time Development of Velocity for Different Values of $\mathrm{Gr}$ in the Case of Impulsive Movement of the Plate

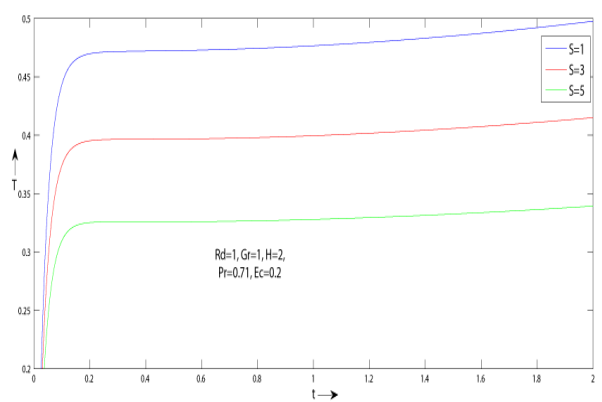

Figure 7: Time Development of Temperature for Different Values of S in the Case of Uniformly Accelerated Movement of the Plate

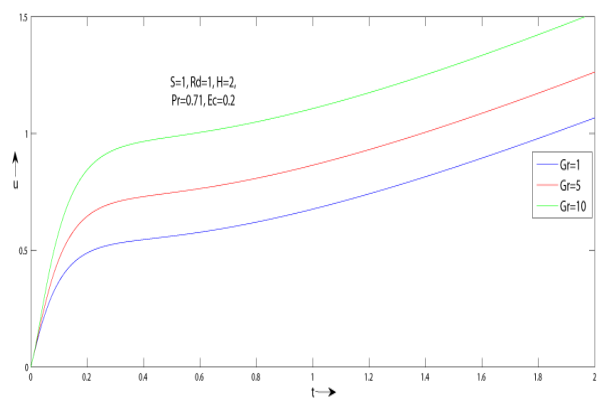

Figure 9: Time Development of Velocity for Different Values of Gr in the Case of Uniformly Accelerated Movement of the Plate

observed in figures 10 and 11 occurs due to the increased viscous and Joule dissipation effects associated with the observed increased velocity.

The effect of the Eckert number $E_{c}$ on the velocity and temperature of the fluid at the centre of the channel $(\mathrm{y}=0.5)$ is shown in figures 12-15. The results show that the fluid velocity and temperature increase with increasing $E_{c}$. Now the Eckert number is proportional to thermal energy dissipation throughout the fluid. Hence, through increased viscous and Joule dissipation effects, an increase in the Eckert number results in the temperature increase observed in figures 12 and 13. The associated increase in flow velocity occurs through free convection currents within the fluid (as shown in figures 14 and 15). 


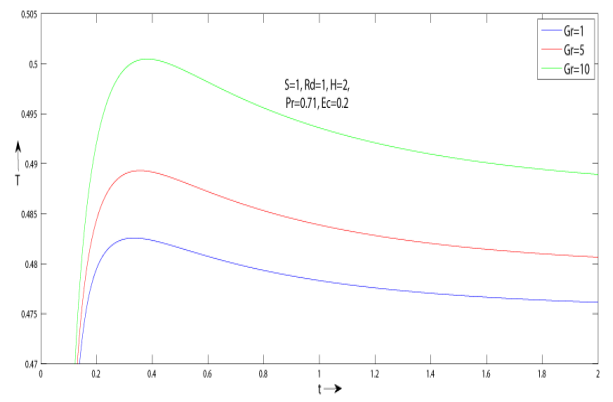

Figure 10: Time Development of Temperature for Different Values of $\mathrm{Gr}$ in the Case of Impulsive Movement of the Plate

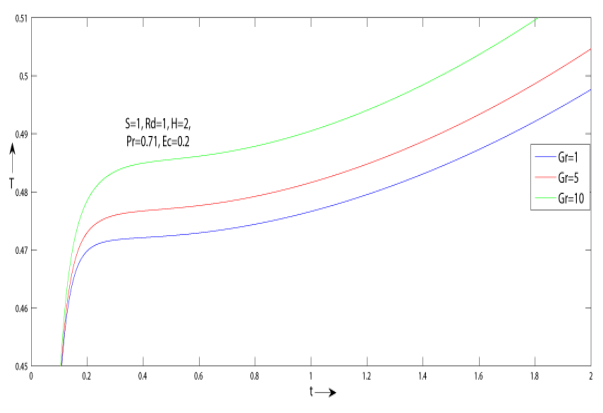

Figure 11: Time Development of Temperature for Different Values of $\mathrm{Gr}$ in the Case of Uniformly Accelerated Movement of the Plate

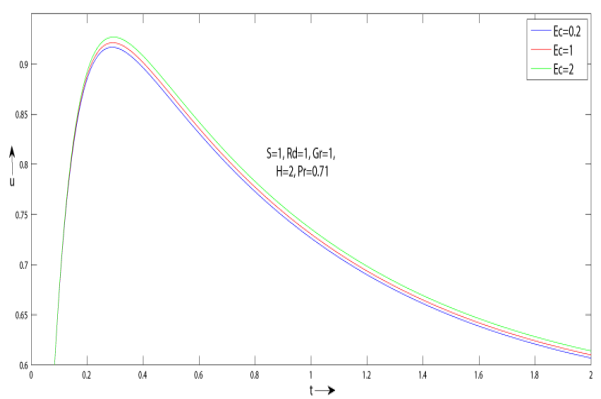

Figure 12: Time Development of Velocity for Different Values of $E_{c}$ in the Case of Impulsive Movement of the Plate

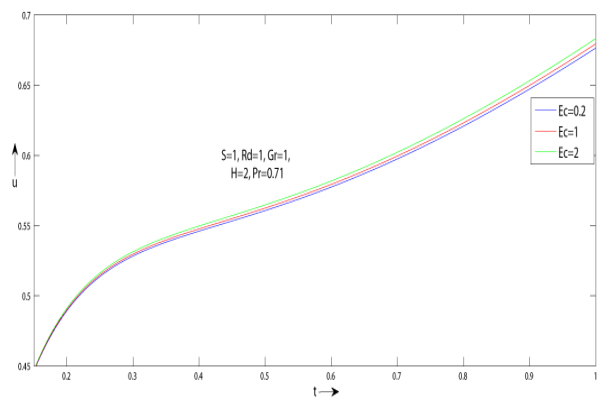

Figure 13: Time Development of Velocity for Different Values of $E_{c}$ in the Case of Uniformly Accelerated Movement of the Plate

The effect of the magnetic parameter $\mathrm{H}$ on the velocity and temperature at the centre of the channel $(\mathrm{y}=0.5)$ is displayed in figures 16-20. Figure 16 revealed that the flow velocity increases with increasing $\mathrm{H}$ in the case of impulsive movement of the plate. An increase in the magnetic parameter causes an increase in the strength of the magnetic field and the associated Lorentz force. The fluid is pulled more strongly by the magnetic field (which is considered to be fixed relative to the moving plate), which results in an increase in the flow velocity. Figure 17 shows that in the case of uniformly accelerated movement of the plate, increasing the magnetic parameter decreases the flow velocity at 


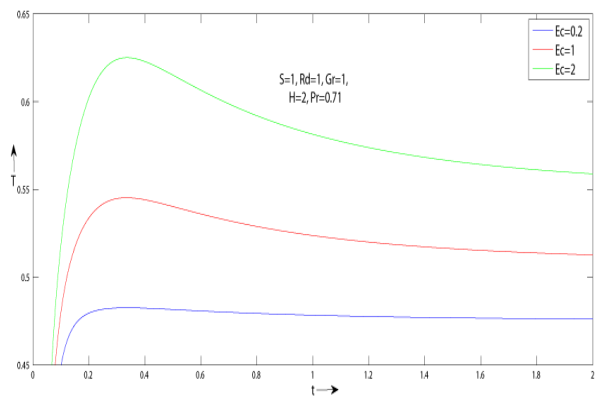

Figure 14: Time Development of Temperature for Different Values of $E_{c}$ in the Case of Impulsive Movement of the Plate

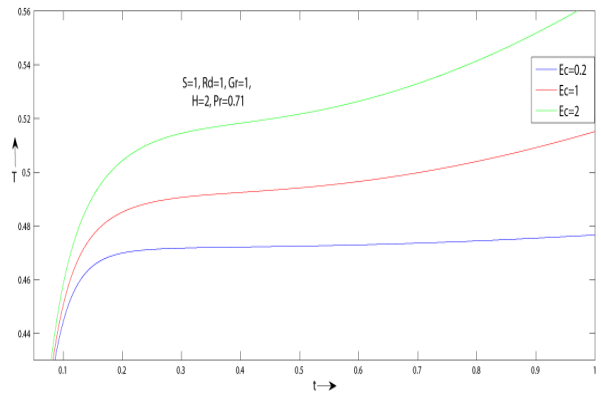

Figure 15: Time Development of Temperature for Different Values of $E_{c}$ in the Case of Uniformly Accelerated Movement of the Plate

small time, but increases it at large time. Near the initial time $t=0$, the velocity of the moving plate is low and the magnitude of the pressure gradient is high. Consequently, the flow velocity at the centre of the channel is greater than the velocity of the plate (as shown in figure 20). Hence, the Lorentz force decelerates the fluid flow at small time. However, as time progresses, the pressure gradient decays, while the Lorentz force on the fluid increases. Thus, the uniformly accelerated movement of the plate dominates the velocity of the fluid over time. Since the magnetic field is fixed relative to the uniformly accelerated plate, the flow is accelerated by the magnetic field at large time. From figures 18 and 19, increasing $\mathrm{H}$ effects an increase in the fluid temperature. The observed temperature increase is caused by Joule dissipation within the fluid, which is proportional to $\mathrm{H}$.

Figures 21-25 show the effects of the radiation parameter $R_{d}$ on the fluid velocity and temperature at the centre of the channel $(y=0.5)$. The results reveal that in the case of the case of impulsive movement of the plate, increasing $R_{d}$ decreases the velocity and temperature of the fluid. If the amount of thermal radiation emitted by the fluid (which may be represented by $R_{d}$ ) is increased, the rate of heat transfer through the fluid is reduced. This accounts for the observed decrease in temperature with increasing $R_{d}$. In the case of uniformly accelerated movement of the plate, increasing $R_{d}$ causes a decrease in the fluid velocity and temperature at small time, but increases them at large time. Since the flow velocity is low near $t=0$, viscous and Joule dissipation effects are negligible at small time. Thus, the increased emission of thermal 


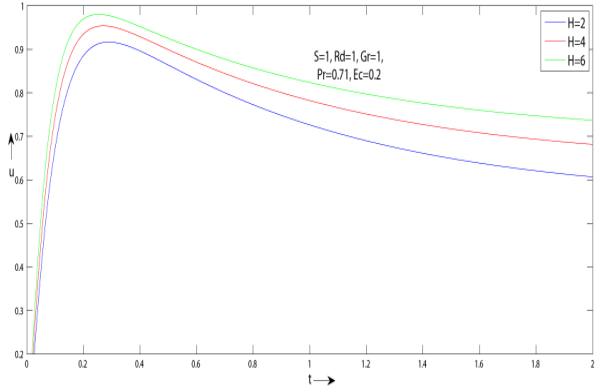

Figure 16: Time Development of Velocity for Different Values of $\mathrm{H}$ in the Case of Impulsive Movement of the Plate

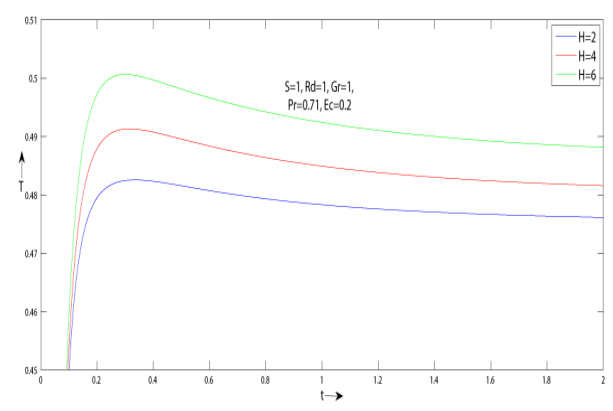

Figure 18: Time Development of Temperature for Different Values of $\mathrm{H}$ in the Case of Impulsive Movement of the Plate

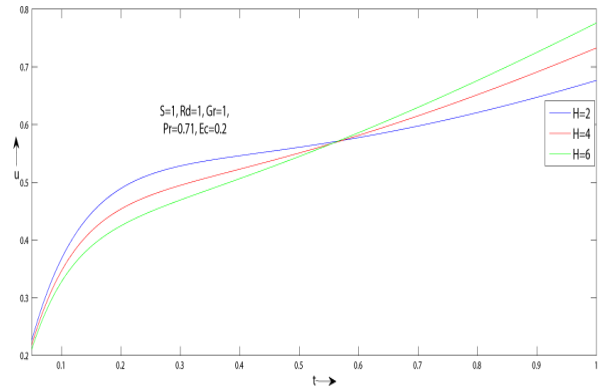

Figure 17: Time Development of Velocity for Different Values of $\mathrm{H}$ in the Case of Uniformly Accelerated Movement of the Plate

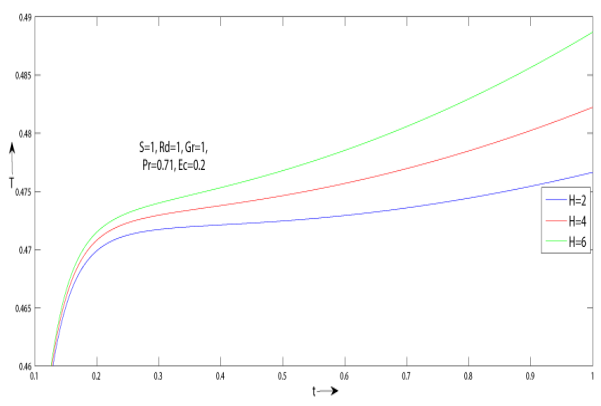

Figure 19: Time Development of Temperature for Different Values of $\mathrm{H}$ in the Case of Uniformly Accelerated Movement of the Plate

radiation associated with increased $R_{d}$ results in a decrease in fluid temperature at small time. This temperature decrease, in turn, causes a reduction in buoyancy forces within the fluid, thereby decreasing the flow velocity. However, since the velocity becomes very large over time due to the uniformly accelerated movement of the plate, the effects of viscous and Joule dissipations have a significant impact on temperature at large times. Therefore, increasing $R_{d}$ increases the temperature of the fluid increases at large time. Through natural convection effects, the flow velocity also increases.

The effects of the Prandtl number Pr on velocity and temperature at the centre of the channel $(y=0.5)$ are shown in figures 26-30. It is observed that 


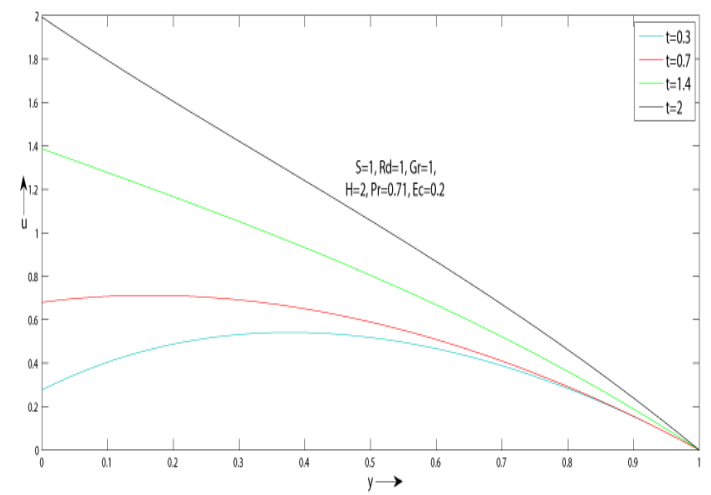

Figure 20: Velocity Profile for Different Values of $t$ in the Case of Uniformly Accelerated Movement of the Plate

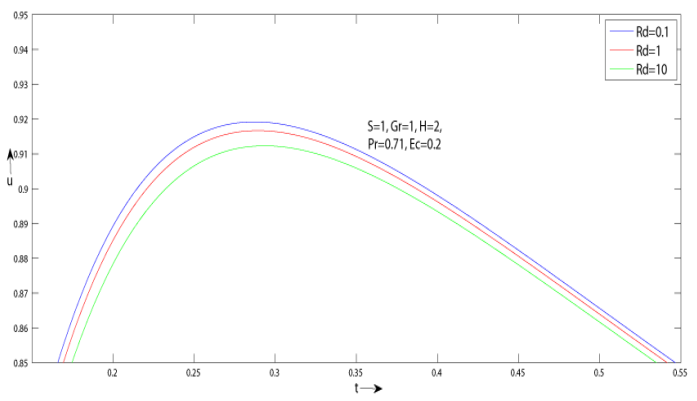

Figure 21: Time Development of Velocity for Different Values of $R_{d}$ in the Case of Impulsive Movement of the Plate

in the case of impulsive movement of the plate, an increase in Pr decreases the velocity and temperature of the fluid. An increase in Prandtl number through a decrease in thermal conductivity effects a decrease in the temperature of the fluid, which also decreases the flow velocity as a result of reduced bouyancy forces. Since the Prandtl number is also proportional to the fluid viscosity, the decrease in flow velocity which occurs with increased Prandtl number is consistent with the expectation that a fluid which has a higher viscosity also has a lower flow velocity. In the case of uniformly accelerated movement of the plate, increasing Pr is found to decrease the fluid velocity and temperature for a short time, but increase them over longer periods of time. Viscous and Joule dissipations may be considered to be negligible near the initial time since the velocity is small. Hence, an increase in Prandtl number causes a decrease in 


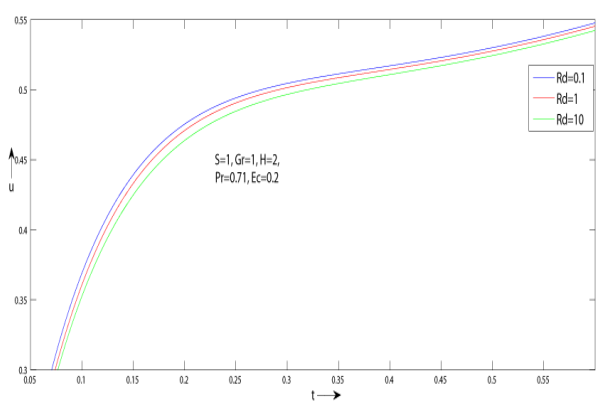

Figure 22: Small-Time Development of Velocity for Different Values of $R_{d}$ in the Case of Uniformly Accelerated Movement of the Plate

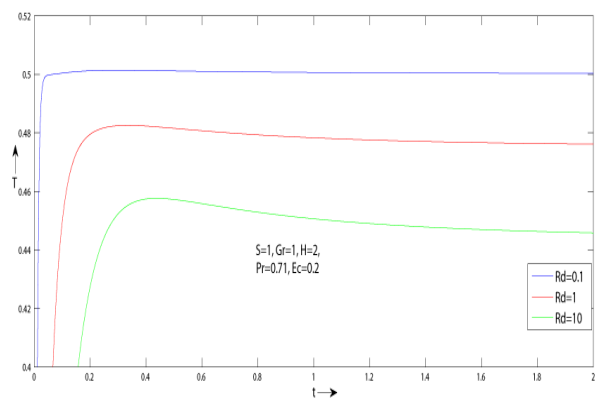

Figure 24: Time Development of Temperature for Different Values of $R_{d}$ in the Case of Impulsive Movement of the Plate

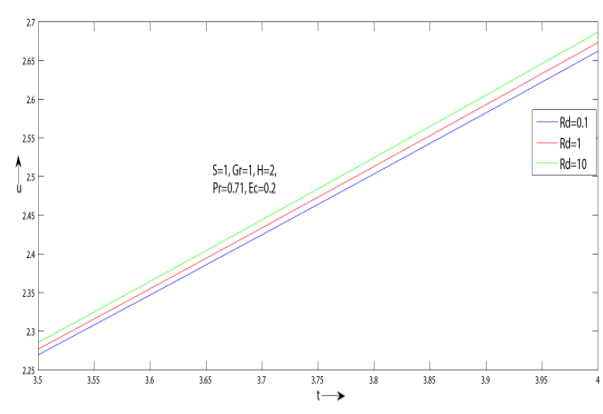

Figure 23: Large-Time Development of Velocity for Different Values of $R_{d}$ in the Case of Uniformly Accelerated Movement of the Plate

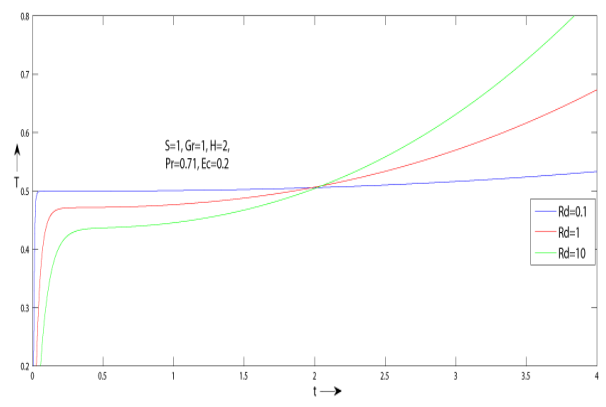

Figure 25: Time Development of Temperature for Different Values of $R_{d}$ in the Case of Uniformly Accelerated Movement of the Plate

fluid temperature at small times, which also decreases the flow velocity due to a reduction in bouyancy forces. On the contrary, viscous and Joule dissipation effects are significant at large time, since the uniformly accelerated movement of the plate causes the flow velocity to become large over time. Therefore, the fluid temperature increases with increasing Pr at large time, which results in an increase in velocity due to the enhanced buoyancy forces. 


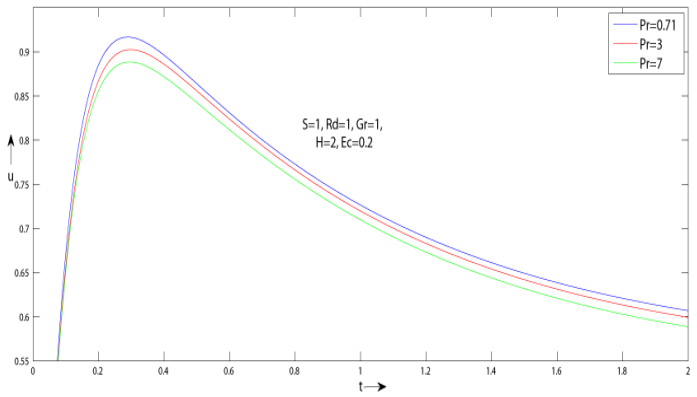

Figure 26: Time Development of Velocity for Different Values of Pr in the Case of Impulsive Movement of the Plate

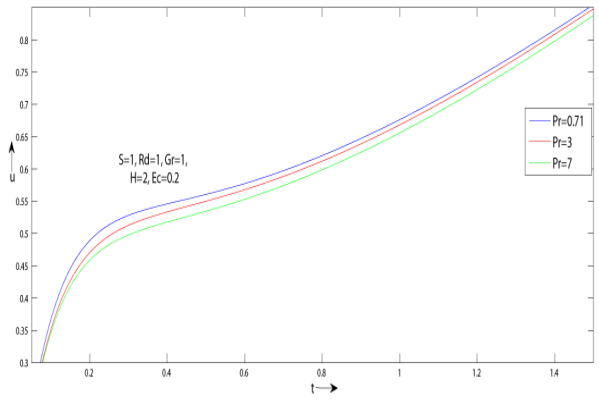

Figure 27: Small-Time Development of Velocity for Different Values of Pr in the Case of Uniformly Accelerated Movement of the Plate

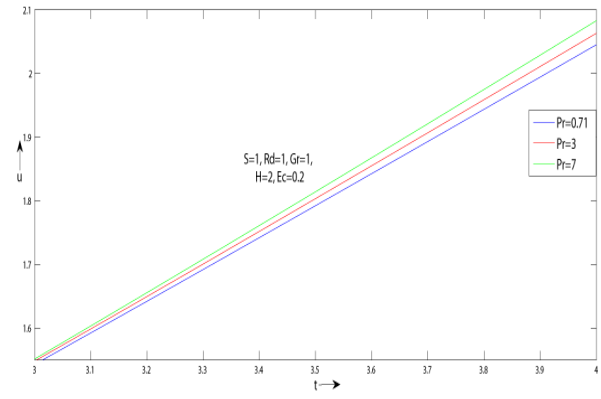

Figure 28: Large-Time Development of Velocity for Different Values of $\mathrm{Pr}$ in the Case of Uniformly Accelerated Movement of the Plate

\section{Conclusion}

In this paper, the unsteady MHD free convection Couette flow of an incompressible viscous fluid between two vertical permeable plates in the presence of thermal radiation and an exponentially decaying pressure gradient was explored. The effects of suction parameter S, radiation parameter Rd, Grashof number Gr, magnetic parameter H, Prandtl number Pr and Eckert number Ec on both the velocity and temperature distributions have been investigated. It was discovered that the radiation parameter, Eckert number and Prandtl number have a greater effect on the temperature than on the velocity. However, the velocity is affected to a greater extent by the Grashof number and 


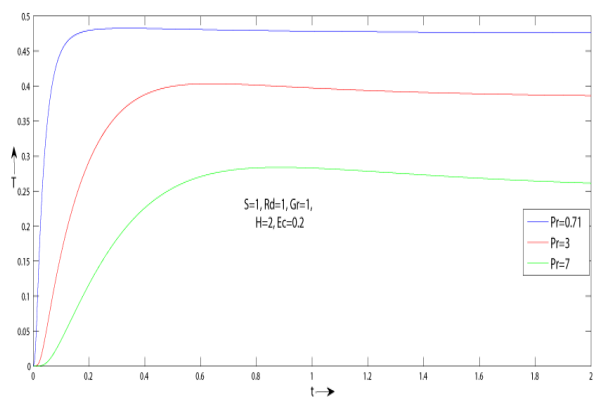

Figure 29: Time Development of Temperature for Different Values of $\mathrm{Pr}$ in the Case of Impulsive Movement of the Plate

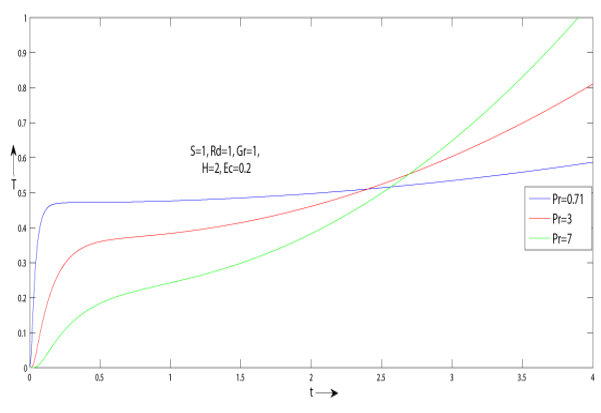

Figure 30: Time Development of Temperature for Different Values of $\mathrm{Pr}$ in the Case of Uniformly Accelerated Movement of the Plate

magnetic parameter. It was discovered that in the case of impulsive movement of the plate, increasing the radiation parameter or Prandtl number causes a decrease in the fluid velocity and temperature. On the other hand, in the case of uniformly accelerated movement of the plate, the radiation parameter and Prandtl number decrease the fluid velocity and temperature at small time but increase them at large time. An increase in the suction parameter was shown to decrease both the velocity and temperature of the fluid, whereas an increase in the magnetic parameter effects an increase in fluid temperature. In the case of impulsive movement of the plate, the magnetic parameter is proportional to the flow velocity. However, increasing the magnetic parameter causes the flow velocity to decrease at small time and increase at large time in the case of uniformly accelerated movement of the plate. The results also revealed that increasing the Grashof number or Eckert number increases the fluid velocity and temperature in both cases.

\section{References}

[1] H.A. Attia, On the Effectiveness of Variation in the Physical Variables on the Generalized Couette Flow with Heat Transfer in a Porous Medium, Research Journal of Physics, 1, No. 1 (2007), 1-9.

[2] H.A. Attia, Unsteady MHD Couette flow with heat transfer in the presence of uniform suction and injection, Engineering Modelling, 22 (2009), 53-56. 
[3] S. Das, M. Jana and R.N. Jana, Couette Flow Through Porous Medium in a Rotating System, International Journal of Mathematical Archive, 2, No. 11 (2011), 2318-2326.

[4] P.A. Davidson, An Introduction to Magnetohydrodynamics, Cambridge University Press, United Kingdom (2001).

[5] S. Eskinazi, Vector Mechanics of Fluids and Magnetofluids, Academic Press Inc., U.S.A. (1967).

[6] A.J.M. Ferriera, Matlab Codes for Finite Element Analysis: Solids and Structures, Springer Science+Business Media B.V., Netherlands (2009).

[7] B.K. Jha, Natural convection in unsteady MHD couette flow, Heat and Mass Transfer, 37 (2001), 329-331.

[8] B.K. Jha and C.A. Apere, Magnetohydrodynamic Free Convective Couette Flow with Suction and Injection, Journal of Heat Transfer, 133, No. 9 (2011), 092501.

[9] V.M. Job and S. Rao Gunakala, Unsteady MHD Free Convection Couette Flow between Two Vertical Permeable Plates in the Presence of Thermal Radiation Using Galerkin's Finite Element Method, International Journal of Mechanical Engineering, 2, No. 5 (2013), 99-110.

[10] V.M. Job and S. Rao Gunakala, Unsteady MHD Free Convection Couette Flow Through a Vertical Channel in the Presence of Thermal Radiation With Viscous and Joule Dissipation Effects Using Galerkin's Finite Element Method, International Journal of Application or Innovation in Engineering $\&$ Management, 2, No. 9 (2013), 50-61.

[11] V.M. Job and S. Rao Gunakala, Unsteady Hydromagnetic Natural Convection Couette Flow through a Vertical Channel in the Presence of Thermal Radiation under an Exponentially Decaying Pressure Gradient with Viscous and Joule Dissipation Effects Using Galerkin's Finite Element Method, International Journal of Application or Innovation in Engineering $\&$ Management, 2, No. 10 (2013), 236-247 .

[12] L.G. Leal, Advanced Transport Phenomena: Fluid Mechanics and Convective Transport Processes, Cambridge University Press, U.S.A. (2007).

[13] M.F. Modest, Radiative Heat Transfer. 3rd ed., Academic Press, Great Britain (2013). 
[14] V.R. Prasad, N.B. Reddy and R. Muthucumaraswamy, Transient radiative hydromagnetic free convection flow past an impulsively started vertical plate with uniform heat and mass flux, Theoret. Appl. Mech., 33, No. 1 (2006), 31-63.

[15] U.S. Rajput and P.K. Sahu, Natural Convection in Unsteady Hydromagnetic Couette Flow Through a Vertical Channel in the Presence of Thermal Radiation, Int. J. of Appl. Math and Mech., 8, No. 3 (2012), 35-56.

[16] J.A. Rao, S. Sivaiah and Sk. Nuslin, Radiation Effects on an Unsteady MHD Vertical Porous Plate in the Presence of Homogeneous Chemical Reaction, ARPN Journal of Engineering and Applied Sciences, 7, No. 7 (2012), 853-859.

[17] J.N. Reddy, An Introduction to the Finite Element Method, McGraw-Hill, U.S.A. (2006).

[18] V.J. Rossow, On Flow of Electrically Conducting Fluids over a Flat Plate in the Presence of a Transverse Magnetic Field, National Advisory Committee for Aeronautics, Technical Note 3971 (1957).

[19] A.K. Singh, Natural Convection in Unsteady Couette Motion, Def. Sci. J., 38 No. 1 (1988), 35-41. 
